\title{
Mass selective infrared spectroscopy using a free electron laser
}

\author{
Mike Putter, Gert von Helden, Gerard Meijer \\ Dept. of Molecular and Laser Physics, University of Nijmegen, Toernooiveld, 6525 ED Nijmegen, The Netherlands \\ Received 31 May 1996
}

\begin{abstract}
Mass selective gas-phase infrared spectroscopy using IR/VUV double resonance ionization is demonstrated. A free electron laser (FEL), tunable over the $100-2000 \mathrm{~cm}^{-1}$ range, is used as the IR excitation source while VUV light, produced by a $F_{2}$ laser, is used for subsequent ionization. Spectra are obtained by monitoring the ion of interest as a function of IR wavelength. It is shown that these spectra can give the same information as direct IR absorption spectra. The sensitivity of the method is many orders of magnitude higher than that of conventional IR absorption spectroscopy. First results on para amino benzoic acid (PABA) are presented.
\end{abstract}

The interaction of light with atoms and molecules is a primary tool for the understanding of atomic and molecular structure. Light in the infrared (IR) spectral region is of particular interest since this is in the energy range of molecular vibrations and it thereby probes the forces holding the atoms in molecules together. The standard technique in the IR is the direct measurement of absorption. A serious limitation to this method is its inherent low sensitivity, although in some cases impressive results have been obtained [1]. In the UV-VIS spectral region, a widely used method to enhance the sensitivity of absorption measurements is to apply laser induced fluorescence (LIF) [2] or resonance enhanced multiphoton ionization (REMPI) [3] techniques. In this way, absorption of only a few photons can be detected, which is clearly impossible to be detected in direct absorption measurements. Some multi-photon fluorescence or ionization studies where one photon is in the IR do exist [4-8]. In addition, Esherick et al. showed greatly improved sensitivity in stimulated Raman spectroscopy by combining this technique with resonant laser ionization $[9,10]$. To obtain information similar to that obtained in direct absorption spectroscopy, a widely tunable IR excitation source as well as a detection method for IR excited molecules that is preferably not vibrational mode specific, is needed. Over the last years free electron lasers (FEL) have become available to users [11-14]. Their performance characteristics make them the ideal light sources for studies of this kind.

In this Letter we present mass selective gas-phase infrared spectroscopy using IR/VUV double resonance ionization. A FEL is used as the IR excitation source while VUV light, produced by a $F_{2}$ laser, is used for subsequent ionization. Spectra are obtained by monitoring the ion of interest as a function of IR wavelength. It is shown that these spectra can give the same information as direct IR absorption spectra. The method is generally applicable to polyatomic molecules and has a sensitivity that is many orders of magnitude higher than that of conventional IR absorption spectroscopy. First results on para amino benzoic acid (PABA) are presented. PABA is chosen 
because its ionization potential (IP) is located about $1000 \mathrm{~cm}^{-1}$ above the photon energy of a $F_{2}$ laser, thereby enabling selective ionization of IR excited levels.

Experiments have been performed at the free-electron-laser user facility FELIX ('free electron laser for infrared experiments') in Nieuwegein, The Netherlands $[14,15]$. This laser produces IR radiation that is continuously tunable over the $100-2000 \mathrm{~cm}^{-1}$ range with a typical bandwidth of $5 \mathrm{~cm}^{-1}$ at $1000 \mathrm{~cm}^{-1}$. In the present experiment FELIX runs at a $5 \mathrm{~Hz}$ repetition rate. The light output consists of so called macropulses of about $3 \mu$ s duration containing $20 \mathrm{~mJ}$ of energy. Each macropulse consists of a train of micropulses which are approximately 3 ps long and $1 \mathrm{~ns}$ apart.

The experimental apparatus consists of a turbopumped vacuum system containing a time-of-flight (TOF) mass spectrometer. The TOF is compact and designed to fulfil second order space focusing conditions [16]. The electrodes are gridless to achieve maximum transmission. There is a $30 \mathrm{~mm}$ distance between the electrodes of the extraction region, a $37 \mathrm{~mm}$ distance between the electrodes of the acceleration region and a $200 \mathrm{~mm}$ drift path between the last electrode and the micro channel plate (MCP) detector. Typical voltages used are $3000 \mathrm{~V}, 1950 \mathrm{~V}$ and ground at the first second and third electrode, respectively. The mass resolution under these conditions is about 200 , limited by the thermal velocity spread of our gas phase sample. Ions are detected on a micro-channel plate (MCP) detector. The ion signal is amplified and recorded on a digital oscilloscope (LeCroy 9430). The data is transferred to a PC for further analysis.

The IR light exits the beam delivery system at FELIX, travels approximately $1 \mathrm{~m}$ through air and enters our experimental apparatus through a $\mathrm{ZnSe}$ window. The IR beam is not focused and has a diameter of about $3 \mathrm{~mm}$. About $1 \mu \mathrm{s}$ after the end of the FELIX macropulse, a $F_{2}$ laser $(158 \mathrm{~nm}$, Lambda Physik EMG 102) is fired. The VUV light travels through an evacuated tube and into the vacuum chamber, counterpropagating the FELIX beam. A circular, $5 \mathrm{~mm}$ diameter, aperture limits the VUV beam diameter and no focusing is applied. The energy in the $10 \mathrm{~ns}$ duration VUV pulse is approximately around $0.1-1.0 \mathrm{~mJ}$ in the ionization region.
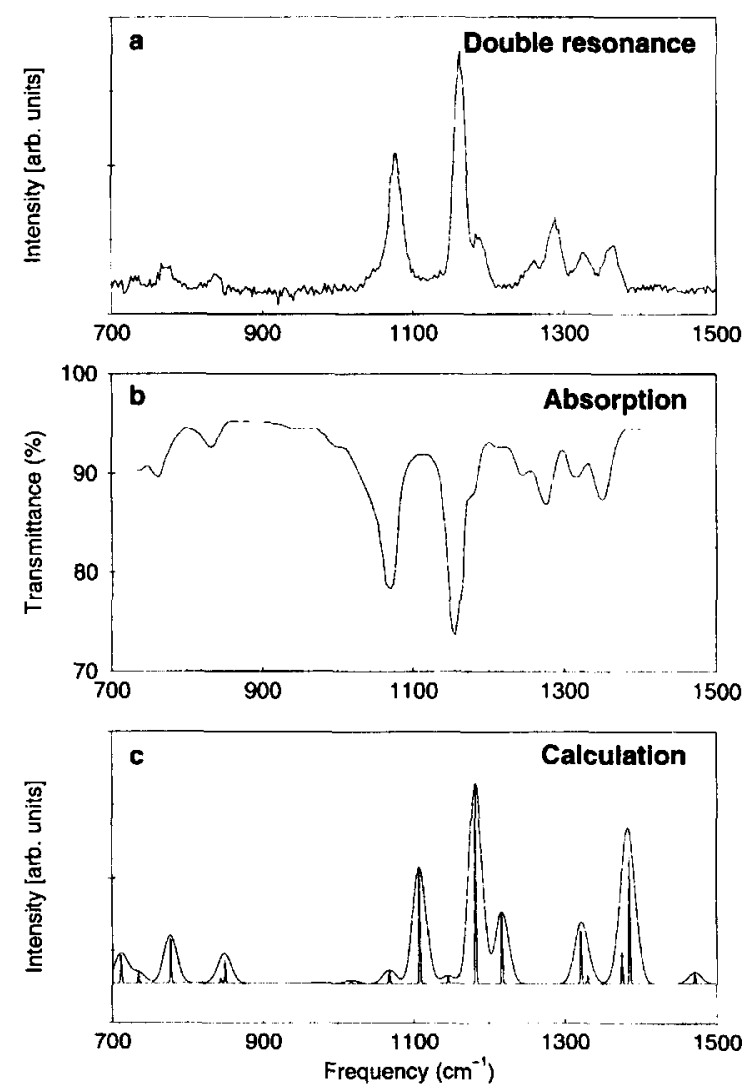

Fig. 1. (a) The measured IR/VUV double resonance spectrum of PABA, (b) the gas phase IR absorption spectrum of PABA taken at $444 \mathrm{~K}$ [18], and (c) the calculated IR absorption spectrum of PABA.

Both beams overlap in the extraction region of the linear TOF setup.

A small amount of solid PABA is placed in the vacuum machine, close to the extraction region of the TOF. The vapor pressure of PABA is calculated using the Clausius-Clapeyron equation and experimental thermodynamic data [17]. At room temperature, the vapor pressure is calculated to be $1.2 \times 10^{-7}$ Torr. This represents an upper bound for the PABA partial pressure in the ionization region since the vacuum chamber is continuously pumped.

In Fig. 1a the signal measured at the mass of the PABA parent ion is shown as a function of IR laser frequency. Several peaks can be identified. The two largest peaks are centered at $1162 \mathrm{~cm}^{-1}$ and $1078 \mathrm{~cm}^{-1}$. A shoulder is observed at $1189 \mathrm{~cm}^{-1}$, on the high frequency side of the main peak. A four 
manifold structure with peaks at $1261 \mathrm{~cm}^{-1}, 1289$ $\mathrm{cm}^{-1}, 1326 \mathrm{~cm}^{-1}$ and $1365 \mathrm{~cm}^{-1}$ is seen. In addition, two weak lines are seen at $773 \mathrm{~cm}^{-1}$ and 842 $\mathrm{cm}^{-1}$. The width of the peaks is wider than the bandwidth of the FELIX radiation by about a factor three.

In Fig. $1 \mathrm{~b}$ the experimental $444 \mathrm{~K}$ gas phase IR absorption spectrum adapted from Kharitonov et al. [18] is shown. All peaks in this spectrum coincide well in position, width and relative intensity with the double resonance spectrum shown in Fig. 1a. Only minor differences are seen. The peaks in Fig. $1 \mathrm{~b}$ all show a small shift to lower frequency of, on the average, $11 \mathrm{~cm}^{-1}$. Also, the peaks in Fig. $1 \mathrm{~b}$ are somewhat broader than the peaks shown in Fig. 1a. Both observations can be attributed to the higher sample temperature used in the direct IR absorption measurements. An elevated temperature will give a wider rotational envelope and therefore broader lines. The frequency shift on the other hand can be explained by anharmonic cross coupling of the IR active modes to thermally excited modes. Due to this effect lines usually shift to lower frequency with increasing temperature [19].

In Fig. 1c the calculated IR absorption spectrum of PABA is shown. The calculations are performed using GAUSSIAN 94 [20] with the Becke3LYP [21] method. Dunning's D95(d, p) [22] basis set has been used. The calculated stick spectrum is convoluted with a Gaussian lineshape with a full width at half maximum of $20 \mathrm{~cm}^{-1}$. No scaling of the calculated frequencies has been done. The agreement between the calculations and the experiment is very good. The disagreement in line positions is less than $1 \%$, a surprisingly small number, especially when considering that Hartree-Fock frequencies are usually calculated too high by more than $10 \%$.

The observation that the IR/VUV double resonance spectrum is so similar to the IR absorption spectrum is not a priori expected. The spectrum shown in Fig. 1a is determined by both the IR absorption and the VUV ionization cross sections. As the VUV ionization cross section will depend on the vibrational level in the ground electronic state, one would expect the double resonance spectrum to be different from the absorption spectrum. The similarity of the two spectra is explained by the influence of intramolecular vibrational energy redistribution
(IVR). Studies on molecules of similar size have shown that IVR takes place on the (sub)-ns time scale in the $1000 \mathrm{~cm}^{-1}$ range [23]. The energy of the absorbed IR photon will therefore be quickly randomized in the molecule and the system will have lost all memory of its initial vibrational state by the time the VUV photons arrive. Ionization will thus take place from many different quantum states, even though a single, well defined state is initially prepared by the IR photon. The total amount of energy in the system is therefore a more important parameter than the initial quantum state.

The IP of jet-cooled PABA in an electric field of $350 \mathrm{~V} / \mathrm{cm}$ has been determined to be $64444 \mathrm{~cm}^{-1}$ [24]. The $F_{2}$ laser emits two lines at 63450 and $63483 \mathrm{~cm}^{-1}$. For a PABA molecule with no internal energy, a minimum IR photon frequency of 961 $\mathrm{cm}^{-1}$ is therefore required to allow subsequent ionization with the $F_{2}$ laser. However, two double resonance peaks are seen below $961 \mathrm{~cm}^{-1}$. In addition, a FELIX independent background PABA ion signal is observed, giving rise to the non-zero baseline in the spectrum shown in Fig. 1a. Both observations are explained by the thermal state distribution of the PABA sample in our experiment.

In Fig. 2a the calculated vibrational density of states of PABA is shown. Vibrational frequencies from the $a b$ initio calculations have been used and the density of states is computed in the harmonic approximation using the Beyer-Swinehart algorithm [25]. In Fig. $2 b$ the room temperature vibrational state distribution is shown. This distribution is obtained via convolution of the density of states with a Boltzmann distribution at $298 \mathrm{~K}$. At room temperature, $55 \%$ of all molecules have an internal energy that is sufficient for ionization by the $F_{2}$ laser. The ionization efficiency as a function of internal energy is unknown, however; it will be zero for internal energies below $961 \mathrm{~cm}^{-1}$ and substantial excess energies might be needed for efficient ionization.

Simultaneous IR multi-photon absorption is not expected to occur under our experimental conditions. This is evidenced by the similarity of the observed double resonance spectrum with the direct absorption spectrum. It is likely, however, that on a strong transition sequential absorption of more than one IR photon per macropulse occurs. From the direct absorption spectrum in Fig. $1 \mathrm{~b}$ a peak absorption cross 



Fig. 2. (a) The calculated vibrational density of states for PABA and (b) the thermal vibrational state distribution at $298 \mathrm{~K}$.

section of $7 \times 10^{-19} \mathrm{~cm}^{2}$ is deduced. Multiplying this with the FELIX fluence per macropulse, approximately $1 \times 10^{19}$ photons $/ \mathrm{cm}^{2}$, leads to an estimated peak absorption of 7 photons per molecule per macropulse. This is possible since the IVR rate is much higher that the photon absorption rate. No saturation effects are seen in the double resonance spectrum. This implies that the ionization efficiency is a steadily increasing function with internal energy.

The IR/VUV double resonance technique presented here is a very sensitive way to obtain gasphase IR absorption spectra of molecules. The method is mass-selective and spectra of pure compounds can be obtained even in mixed samples. In the present experimental setup the IR laser beam diameter is $3 \mathrm{~mm}$ and the acceptance length of the TOF electrodes is $1 \mathrm{~cm}$, so a volume of $0.07 \mathrm{~cm}^{3}$ is probed. This corresponds to only $3 \times 10^{8} \mathrm{PABA}$ molecules, using a room temperature vapor pressure of $1.2 \times 10^{-7}$ Torr, which should be compared to the pressure of 1.4 Torr and the path length of $10 \mathrm{~cm}$ that is used in the direct IR absorption experiment
[18]. This means an increase in sensitivity of about eight orders of magnitude. Increasing the efficiency in ionization and ion detection can yield an even further increase in sensitivity. It seems realistic that the IR absorption spectra of molecules at densities of $10^{5}$ molecules $/ \mathrm{cm}^{3}$, corresponding to a partial pressure below $10^{-11}$ Torr, can be measured. Modern methods of tunable VUV generation should make this method applicable to a variety of molecules as well as to interesting species such as radicals or clusters where IR spectroscopic information could so far not be obtained.

\section{Acknowledgements}

We gratefully acknowledge the support by the 'Stichting voor Fundamenteel Onderzoek der Materie' (FOM) in providing the required beam time on FELIX and highly appreciate the skilful assistance by the FELIX staff, in particular Dr. A.F.G. van der Meer. We would like to thank E.G.H. van Leeuwen without whom the experimental apparatus would never have been completed in time. This work has become possible by financial support by the Netherlands Organisation for Scientific Research (NWO) via PIONIER-grant \#030-66-89.

\section{References}

[1] T.F. Giesen, A. Vanorden, H.J. Hwang, R.S. Fellers, R.A. Provencal and R.J. Saykally, Science 265 (1994) 756.

[2] A. Schultz, H.W. Cruse and R.N. Zare, J. Chem. Phys. 57 (1972) 1354.

[3] M.N.R. Ashfold and J.D. Howe, Ann. Rev. Phys. Chem. 45 (1994) 57.

[4] A. Seilmeier, W. Kaiser, A. Laubereau and S.F. Fischer, Chem. Phys. Letters 58 (1978) 225.

[5] P. Esherick and R.J.M. Anderson, Chem. Phys. Letters 70 (1980) 621.

[6] T. Seelemann and P. Andresen, Chem. Phys. Letters 146 (1988) 89.

[7] R.H. Page, Y.R. Shen and Y.T. Lee, J. Chem. Phys. 88 (1988) 4621.

[8] D. Feldmann, J. Laukemper and K.H. Welge, J. Chem. Phys. 79 (1983) 278.

[9] P. Esherick, A. Owyoung and J. Pliva, J. Chem. Phys. 83 (1985) 3311.

[10] P. Esherick and A. Owyoung, Chem. Phys. Letters 103 (1983) 235. 
[11] E.B. Szarmes, A.D. Madden and J.M.J. Madey, Nucl. Instr. Methods A 358 (1995) 220.

[12] A. Marziali, T.I. Smith and H.A. Schwettman, Nucl. Instr. Methods A 358 (1995) 252.

[13] D.A. Jaroszynski, R. Prazères, F. Glotin and J.M. Ortega, Phys. Rev. Letters 72 (1994) 2387.

[14] D. Oepts, A.F.G. van der Meer and P.W. van Amersfoort, Infrared Phys. Technol. 36 (1995) 297.

[15] G.M.H. Knippels, R.F.X.A.M. Mols, A.F.G. van der Meer, D. Oepts and P.W. van Amersfoort, Phys. Rev. Letters 75 (1995) 1755.

[16] R. Weinkauf, K. Walter, C. Weickhardt, U. Boesl and E.W. Schlag, Z. Naturforsch. a 44 (1989) 1219.

[17] R. Sabbah, R. Chastel and M. Laffitte, Can. J. Chem. 52 (1974) 2201.

[18] I.I. Kharitonov and I.I. Oleinik, Dokl. Akad. Nauk SSSR 313 (1990) 384.
[19] G. Herzberg, Molecular spectra and molecular structure II, infrared and Raman spectra of polyatomic molecules (van Nostrand-Reinhold Inc., New York, 1945).

[20] GAUSSIAN 94 (Revision B.3), M.J. Frisch et al., Gaussian, Inc., Pittsburgh PA (1995).

[21] A.D. Becke, J. Chem. Phys. 98 (1993) 5648.

[22] T.H. Dunning, Jr. and P.J. Hay, in Modern Theoretical Chemistry, Ed. H.F. Schaefer, III, (Plenum, New York, 1976).

[23] P.M. Felker, W.R. Lambert and A.H. Zewail, J. Chem. Phys. 82 (1985) 3003.

[24] G. Meijer, M.S. de Vries, H.E. Hunziker and R.H. Wendt, J. Chem. Phys. 92 (1990) 12.

[25] S.E. Stein and B.S. Rabinovitch, J. Chem. Phys. 58 (1973) 2438. 